\title{
Ultra-luminous X-ray Sources
}

\author{
Martin Ward \\ Department of Physics, University of Durham, Durham, UK \\ email: m.ward@durham.ac.uk
}

\begin{abstract}
ULXs have been studied for 20 years, but only recently have detailed X-ray spectral/timing studies, and investigations of their statistical properties and their environments and counterparts been undertaken. I will review progress in these areas.
\end{abstract}

Keywords. X-rays: Ultra-luminous sources, starformation.

\section{Introduction}

Ultra-luminous X-ray sources (ULXs) are a sub-set of X-ray sources which emit $L_{\mathrm{X}}>$ $10^{39} \mathrm{erg} \mathrm{s}^{-1}$, and can reach luminosities of up to $10^{41} \mathrm{erg} \mathrm{s}^{-1}$. They are so-called because these luminosities significantly exceed those of known discrete X-ray sources within our Galaxy and M31. Note: the X-ray luminosities quoted for ULXs are always broad-band, but the actual energy range can differ from study to study, and so comparisons need to be made with some care, particularly if the range used extends to low energies, and for sources with soft excesses. Symmetric accretion onto a $1.4 \mathrm{M}_{\odot}$ compact object with $L_{\mathrm{X}}>10^{39} \mathrm{erg} \mathrm{s}^{-1}$ implies a substantially super-Eddington accretion rate. Or, if the compact object is emitting at the Eddington limit then the black hole must be 10's of solar masses. The situation of anisotropic emission will be considered later. Under the standard assumptions the highest mass black hole that can be formed in a galactic binary system is $\sim 20 \mathrm{M}_{\odot}$ (see Fryer \& Kalogera 2001), which is close to that observationally determined in the case of a galactic micro-quasar (GMQ). For super-massive black holes (SMBHs) at the centres of galaxies, the lowest mass estimated is in the range $\sim 10^{4}$ to $10^{5} \mathrm{M}_{\odot}$, Filippenko and Ho (2003). From this is it clear that there are about three orders of magnitude between the highest mass black hole in a binary system, and the lowest mass black hole at the centre of a galaxy. It is natural therefore that studies of ULXs have become inextricably linked to the search for intermediate mass black holes (IMBHs). For completeness one should note that globular clusters have been proposed as hosts for IMBHs with masses of $\sim 1000$ 's $\mathrm{M}_{\odot}$, but previous claims to have detected such objects should be treated with caution (Gerssen et al. 2003). In this review I will discuss the multi-frequency, temporal and environmental properties of ULXs with particular attention to the two main models proposed to explain their nature; that they are IMBHs or alternatively that they are variations on the types of X-ray binary system that we have identified within our Galaxy.

\subsection{A brief history of $U L X s$}

ULXs have been known of observationally since the early 1980's, although they were not originally referred to using this acronym. In a study of M51 using the Einstein Observatory Palumbo et al. (1985) suggested that the brightest extra-nuclear sources have compact object masses of $\sim 10 \mathrm{M}_{\odot}$. In a comprehensive review, Fabbiano (1989) pointed out that such sources are quite common in X-ray studies of nearby galaxies. Later 
observations of a dwarf star forming galaxy, NGC5408, led Fabian and Ward (1993) to suggest a mass of the accreting object of $\sim 100 \mathrm{M}_{\odot}$. There followed statistical studies of galaxy samples, e.g. Roberts and Warwick (2000), who showed that in a ROSAT sample one in five galaxies contained at least one source with $L_{\mathrm{X}}>10^{39} \mathrm{erg} \mathrm{s}^{-1}$. The Chandra era, with its sub-arcsecond imaging capability, drastically reduced the problem of source confusion, and contamination from extended diffuse emission. One of the early Chandra results was the identification of an off-nucleus highly variable X-ray source in M82, which reached a luminosity of more than $10^{41} \mathrm{erg} \mathrm{s}^{-1}$; Kaaret et al. (2001), Matsumoto et al. (2001). The ULX in M82 is a single spectacular example, but the best studied ensemble of ULXs are those found in the Antennae system, which are the subject of a very detailed analysis, see Fabbiano et al. (2003) and references therein. In the remainder of this review I will focus on specific results that have bearing on the nature of ULXs.

\section{Models of ULXs}

If we adopt the most straightforward interpretation of the Eddington limit, then the highest luminosity ULXs require accretion onto a compact object of $\sim 1000 \mathrm{M}_{\odot}$, or much more if the accretion process is inefficient at radiating energy, i.e. an ADAF. At the other extreme it is possible that the emission is really super-Eddington, resulting from radiatively driven inhomogeneities in the accretion disc, as propsed by Begelman (2002). Alternatively, if the emission is anisotropic which could result from thermal timescale mass-transfer (King et al. 2001), this could explain the luminosity of an average ULX. More extreme beaming is present in galactic micro-quasars, and a link between ULXs and these objects was first proposed by Reynolds et al. (1997) for Dwingeloo X-1, and in more detail by Kording et al. (2002). It is these generic models that the studies described below aim to confront.

\section{X-ray Properties of ULXs}

Because the definition of ULXs is based on a single parameter, its luminosity, it is probable that the class will be heterogeneous, and in particular may contain examples of both high and low mass X-ray binaries, and some supernova remnants. In order to focus the discussion I will exclude ULXs that are positively identified with SNRs, and also those found in early type and elliptical galaxies. Irwin et al. (2004) have studied the properties of ULXs suggested to be associated with early type galaxies, and find their number consistent with that expected for background sources. This means the incidence of ULXs in ellipticals and S0s is consistent with zero. In addition, no ULX at the upper end of the luminosity distribution has so far been found in an elliptical. These facts point to any confirmed (non-background) ULX in early type galaxies being associated with the high luminosity end of the low-mass X-ray binary population. In the following sections I will discuss results for ULXs associated with spiral and starforming galaxies.

\subsection{X-ray Spectra}

In standard accretion discs the temperature is inversely related to the black hole mass as $L \propto M^{-1 / 4}$. This means that if IMBHs exist in ULXs their X-ray spectra should be fitted by cooler accretion discs than those found in stellar-mass black hole candidates. Based on good quality X-ray spectra of a number of ULXs at the higher end of the luminosity distribution $L_{\mathrm{X}}>10^{40} \mathrm{erg} \mathrm{s}^{-1}$, Miller et al. (2004) show that their disc temperatures are 5-10 times lower than found in Galactic stellar mass black hole systems in their high states. This would imply black holes in these ULXs of up to a few $1000 \mathrm{M}_{\odot}$. 
However, as the authors point out the spectral complexity at soft X-ray energies is not well understood in these sources, and if it does not arise from a disc as assumed, but rather from an optically thin thermal plasma, then the black hole mass determinations are incorrect. However, no emission lines characteristic of a thermal plasma have so far been detected, supporting the cool disc hypothesis. It is somewhat disappointing to see that even given good signal/noise X-ray spectra of some ULXs, e.g. NGC 5204 X-1, and HoII X-1, Roberts et al. (2005) and Goad et al. (2005), the models still result in ambiguity in distinguishing between stellar mass and intermediate mass black holes. It is likely that we need a more sophisticated understanding of the soft energy components and the role of jets and winds, as well as better data at hard energies to constrain power-law versus multi-colour disc models.

\subsection{X-ray Variability}

Perhaps the most obvious and secure use of high amplitude X-ray variability data for ULXs, is to rule out the presence of multiple unresolved sources, and an SNR origin of the X-rays.

There are many X-ray variability studies of ULXs, and I will highlight results for just a few of the best studied cases. The ULX in M82 is at the high end of the luminosity distribution with an X-ray luminosity up to $10^{41} \mathrm{erg} \mathrm{s}^{-1}$. Strongly beamed X-rays are ruled out in this source by the presence of a QPO, thought to be related to disc oscillations, and the detection of iron K alpha emission, Strohmayer \& Mushotzky (2003). This is because a significant beamed component would have diluted the QPO amplitude and $\mathrm{K}$ alpha emission, making these features difficult or impossible to detect. Unfortunately $\mathrm{X}$-ray spectral fitting is not very useful for this source because of the column density and significant contamination from an extended diffuse plasma component. In another example, NGC4559 X-7, Cropper et al. (2004) have identified a break in the power density spectrum. In the case of SMBHs and stellar mass BHs there are often two breaks in the power density spectrum, and so the break in NGC4559 X-7 is consistent with either $38 \mathrm{M}_{\odot}$ or $1300 \mathrm{M}_{\odot}$. Another impressive case of a quasi period in a ULX is seen in M74(NGC628) X-1, see Liu et al. (2005), and Krauss et al. (2005). This source exhibits extreme variablity on timescales of a few 1000 seconds. Once again any mass estimate depends strongly on the chosen interpretation of the QPO.

So it seems at present that the detection of QPOs and power density breaks can be useful for constraining the effects of beaming, but not as a reliable direct mass determination.

\section{The Environment of ULXs}

The environments of ULXs may be conveniently separated into three topics; their global environment, i.e. the host galaxy, their local environment within the host galaxy, e.g. associated nebulae, star clusters etc. and finally direct association with an optical or compact radio counterpart.

\subsection{The Host Galaxy}

It is evident that the ULX phenomenon is associated with star formation. A spectacular example being the Antennae system which contains 9 ULXs. At higher redshift the Cartwheel starforming ring contains 20 ULXs, although without variablity data we cannot exclude the possiblity that some sources are multiple but not spatially resolved, Gao et al. (2003) and Wolter and Trinchieri (2004). It is proposed by Grimm et al. (2003), that there exists a universal X-ray luminosity function (LF) for high mass X-ray binaries, 
if one normalizes each separate LF by the appropriate star formation rate for the galaxy concerned. Wolter and Trinchieri find that the LF for the Cartwheel fits this universal function, but with a factor 5 higher cut-off in $L_{\mathrm{X}}$. This may be connected with the fact that ULX occurrence is related to galaxy type (and/or metal abundance) since they are often found in nearby dwarf galaxies.

\subsection{The Local Environment}

The more luminous ULXs are frequently located near to sites of active star formation such as super star clusters containing $10^{6} \mathrm{M}_{\odot}$ of stars within a volume only a few parsecs across. It is suggested that encounters within such a high stellar density environment could result in a high fraction of binaries. An interesting result found by Kaaret et al. (2004b), is that there is often a slight offset between the position of the ULX and a nearby star cluster. This could be interpreted as resulting from the ULX being ejected from the cluster via dynamical interactions. The closeness to the location of birth assumed to be within the cluster, means that the ULX phenomenon switches on soon after their formation. There are a number of ULX that are cospatial with emission nebulae, and spectroscopy and imaging of these nebulae have been the subject of intensive effort over the last few years, (see contribution from Manfred Pakull in these proceedings). Some of these nebulae are very large, e.g. 400pc across for NGC1313 X-2, Pakull and Mirioni (2002), and 200pc across for IC 342 X-1, Roberts et al. (2003). The energies required to produce the emission lines and to drive the expansion velocities, are higher than those found in a typical supernova, and so multiple supernova events have been suggested, or evidence of a hypernova event.

Generally the optical emission lines from these nebulae are consistent with those seen in SNRs. However, in the case of HoII the spectrum has a strong line from HeII, Pakull and Mirioni (2002). They used the spectrum to estimate the luminosity required to photoionise the nebula. Kaaret, Ward and Zezas (2004) observed HoII using the HST to provide images in several emission lines including HeII, and derived a photoionised luminosity for the nebula consistent with that of Pakull and Mirioni. This implies that the detected X-rays are not significantly beamed, and so the black hole should have a mass in the range $\sim 25$ to $40 \mathrm{M}_{\odot}$.

\subsection{Optical Counterparts}

The search for optical counterparts to ULXs is hampered by their association with star forming regions. This often means that the fields are crowded, so that accurate astrometry is essential. By alignment of the position of an X-ray source in the field with a known counterpart, such as a SNR, it is possible to achieve relative astrometry between the $\mathrm{X}$-ray and optical of a few tenths of arcseconds. Table 1 summarises the information on the five best candidates for optical counterparts of ULXs. They are all young stars, consistent with the connection between ULXs and starformation. These counterparts are all predictably faint, but in the case of Holmberg II, HST/STIS spectroscopy was obtained by Liu et al. (2004). The spectrum contained the high ionisation emission line of NV, strongly supporting this star as the X-ray source counterpart. Similarly Kuntz et al. (2005) detected spatially unresolved HeII and HeI emission lines at the position of the counterpart to M101 X-1, confirming the physical association with the X-ray source. No ULX has so far been observed to eclipse, but there is the possiblity of obtaining a direct measurement of the black hole mass function via determination of an orbital period and the orbital velocities. This would be extremely challenging currently for such faint sources, but could be viable in the era of extremely large telescopes. 
Table 1. Optical Counterparts to ULXs

\begin{tabular}{lcc}
\hline Source & Spectral Type & Reference \\
\hline M81 X-11 & O8V & Liu et al. $(2002)$ \\
NGC1313 & OV-O-BI & Zampieri et al. $(2004)$ \\
Holmberg II & O4V-B3 Ib & Kaaret et al. (2004) \\
NGC5204 & B0 Ib & Liu et al. $(2004)$ \\
M101 & mid-B supergiant & Goad et al. $(2002)$ \\
\end{tabular}

\subsection{Radio Observations of ULXs}

Given that X-rays dominate the energy output of ULXs, it might not seem obvious that radio observations would prove a fruitful band to employ in the study of these objects. However, there are two main reasons why radio observations are potentially very useful. First, given the strong evidence that ULXs are related to starformation, they may be direcly or indirectly associated with supernovae and their remnants. This likelihood increases for sources with lower X-ray luminosities, soft X-ray spectra and with no evidence of X-ray variability. An example is SN1987K in NGC1313, Schlegel et al. (2000). In relatively rare cases some young SNR can reach X-ray luminosities in excess of $10^{40} \mathrm{erg} \mathrm{s}^{-1}$, Fox et al. (2000), also see the contribution of Norci and Meurs in these proceedings. Such examples are clearly interesting in their own right, but they do not help us to address questions regarding intermediate mass black holes. There are now several examples of ULXs previously identified with radio bright SNRs, that based on their X-ray variability are now re-classified as compact binaries eg. the radio hypernova remnants in M101 (Snowden et al. 2001) and the source M16 in NGC6969 (Roberts and Colbert 2003) are now claimed to be X-ray binaries. Furthermore, the radio and optical emission line nebulae around some ULXs may arise from the SNR associated with the birth of the ULX, for example IC342 X-1, Roberts et al. (2003), and M81 X-9, Wang (2002). The second reason for radio studies is that if radio emission is detected it might be intrinsic to the ULX, and hence provide a direct probe of the physics of the ULX phenomena. The first suggestion of this is the radio detection of the ULX in NGC5408, for which it is claimed the emission may be beamed in a way analogous to that in the galactic micro-quasars (GMQs), Kaaret et al. (2003). However, a second epoch radio observation of NGC5408 reported by Kording et al. (2005), shows the emission to be consistent with a steady source, unlike the typical radio properties of GMQs. Radio emission has also been detected from the ULX in the dwarf galaxy Holmberg II, Miller et al. (2005). In this case it is extended by about $60 \mathrm{x} 40 \mathrm{pc}$, and is cospatial with the optical HeII line nebula. The optical and radio properties argue against the radio arising from an HII region or a SNR, and Miller et al. suggest that we may be seeing the same phenomena as in the W50 nebula surrounding the jet source in SS433, but with 10 times greater energy within the nebula.

To establish a link between radio emission from ULXs and galactic micro-quasars requires radio monitoring, since the beamed radio emission from GMQs is highly variable. Intriguingly one of the most luminous and best studied of all ULXs, that in M82, is spatially coincident with a transient radio source, detected only once (Kording et al. 2005). This could be interpreted as evidence in favour of a radio outburst in a beamed GMQ, but it is also consistent with a faded radio supernova, which might be related to the birth of the ULX. Clearly any future reappearance of the radio emission would be a critical test of the beaming model. 


\section{Conclusions}

ULXs are undoubtably a heterogeneous class, but the variable high luminosity examples, $L_{\mathrm{X}}>10^{40} \mathrm{erg} \mathrm{s}^{-1}$, may be more homogeneous, and are the likely candidates for IMBHs. The association between ULXs and starformation is proven, although their formation and duty cycle in different spectral states remain open questions. The issue of anistropic emission/beaming has been investigated by studies of their associated nebulae, but so far this has not resulted in mass estimates of more than a few $10^{\prime} s \mathrm{M}_{\odot}$. Improved understanding of both their soft and hard X-ray spectral components will help in deciding whether a cool accretion disc, hence high $\mathrm{BH}$ mass, is the correct interpretation.

\section{References}

Begelman, M. 2002, ApJ 568. L97

Cropper, M., et al. 2004, MNRAS 349, 39

Fabbiano, G. 1989, A.Rev.Astron.Astrophys. 27, 87

Fabbiano, G., et al. 2003, ApJ 584, L5

Fabian, A.C. \& Ward, M.J. 1993, MNRAS 263, L51

Filippenko, A.V. \& Ho, L.C. 2003, ApJ 588, L13

Fox, D.W., et al. 2000, MNRAS 319, 1154

Fryer, C.L. \& Kalogera, V. 2001, ApJ 554, 548

Gao, Y., et al. 2003, ApJ 596, L171

Grimm, H.-J., Gilfanov, M., \& Sunyaev, R. 2003, MNRAS 339, 793

Gerssen, J., van der Marel, R.P., Gebhardt, K., \& Guhathakurta, P. 2003, AJ 125, 376

Goad, M,R., Roberts, T.P., Knigge, C., \& Lira, P. 2002, MNRAS 335, L67

Goad, M.R., Roberts, T.P., Reeves, J., \& Uttley, P. 2005, astro-ph/ 0510185

Irwin, J.A., Bregman, J.N., \& Athey, A.E. 2004, ApJ 601, L143

Kaaret, P., et al. 2001, MNRAS 321, L29

Kaaret, P., et al. 2003, Science 299, 365

Kaaret, P., Ward, M.J., \& Zezas, A. 2004, MNRAS 351, L83

Kaaret, P., et al. 2004b, MNRAS 348, L28

King, A., et al. 2001, ApJ 552, L109

Kording, E., Falcke, H., \& Markoff, S. 2002, Astron. ES Astrophys 382, L13

Kording, E., Colbert, E., \& Falcke, H. 2005, Astron. E Astrophys 436, 427

Krauss, M.I., et al. 2005, ApJ 630, 228

Kuntz, K.D., et al. 2005, ApJ 620, L31

Liu, J-F., et al. 2002, ApJ 580, L31

Liu, J-F., Bregman, J.N., \& Seitzer, P. 2004, ApJ 602, 249

Liu, J-F., et al. 2005, ApJ 621, L17

Miller, N.A., Mushotzky, R.F., \& Neff, S.G. 2005, ApJ 623, L109

Miller, J.M., Fabian, A.C., \& Miller, M.C. 2004, ApJ 607, 931

Matsumoto, H., et al. 2001, ApJ 547, L25

Pakull, M.W. \& Mirioni, L. 2002, ESA SP-488, astro-ph/ 0202488

Palumbo, G., et al. 1985, ApJ 298, 259

Reynolds, C.S., et al. 1997, MNRAS 286, 349

Roberts, T. P. \& Warwick, R.S. 2000, MNRAS 315, 98

Roberts, T.P. \& Colbert, E.J.M. 2003, MNRAS 341, L49

Roberts, T.P, Goad, M.R., Ward, M.J., \& Warwick, R.S. 2003, MNRAS 342, 709

Roberts, T.P., et al. 2005, MNRAS 357, 1363

Schlegel, E.M., et al. 2000, ApJ 120,791

Strohmayer, T.E. \& Mushotzky, R.F. 2003, ApJ 586, L61

Wang, D.Q. 2002, MNRAS 332, 764

Wolter, A. \& Trinchieri, G 2004, Astron. \& Astrophys. 426, 787

Zampieri, L., et al. 2004, ApJ 603, 523 


\section{Discussion}

Bosch-Ramon: Comment: A way for distinguishing ULXs with intermediate BH and strongly Doppler boosted $\mu \mathrm{Q}$ jets could be the presence of radio emission: lacking in intermediate $\mathrm{BH}\left(\mathrm{L}_{R} \propto \mathrm{L}_{X}{ }^{0.7}\right)$ and probably present in strongly Doppler boosted $\mu \mathrm{Q}$ jets.

WARD: Radio observations are certainly a valuable tool in the multi-frequency study of ULX. One problem is ambiguity - if detected, is the radio related to a Supernovae event or a microquasar? Clearly spectral index and temporal monitoring will help answer this question.

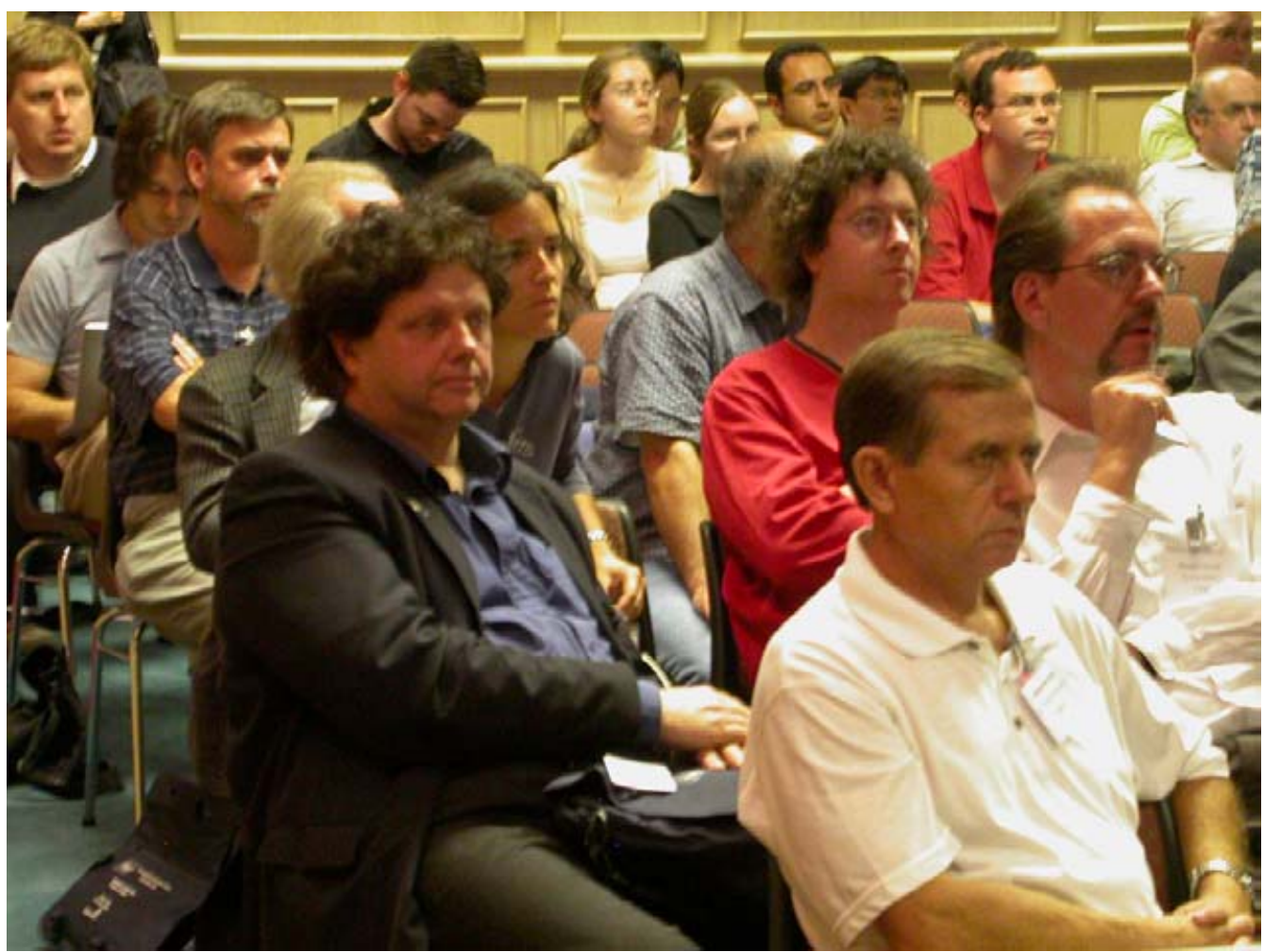

Dr Who in the audience. 